Bull. Korean Math. Soc. 49 (2012), No. 3, pp. 495-510

http://dx.doi.org/10.4134/BKMS.2012.49.3.495

\title{
CONVERGENCE RATES FOR THE MOMENTS OF EXTREMES
}

\author{
Zuoxiang Peng and Saralees Nadarajah
}

\begin{abstract}
Let $X_{1}, X_{2}, \ldots, X_{n}$ be a sequence of independent and identically distributed random variables with common distribution function $F$. Convergence rates for the moments of extremes are studied by virtue of second order regularly conditions. A unified treatment is also considered under second order von Mises conditions. Some examples are given to illustrate the results.
\end{abstract}

\section{Introduction}

Let $\left(X_{n}\right)$ be a sequence of independent and identically distributed random variables with common distribution function $F$. Let $M_{n}=\max \left\{X_{1}, X_{2}, \ldots\right.$, $\left.X_{n}\right\}$ denote the partial maximum of $\left(X_{n}\right)$. Suppose that $F$ is in the domain of attraction of an extreme-value distribution

$$
G(x)=G_{\gamma}(x)=\exp \left\{-(1+\gamma x)^{-\frac{1}{\gamma}}\right\}, \quad \gamma \in \mathbb{R}, \quad 1+\gamma x \geq 0,
$$

which means that there exists normalizing constants $a_{n}$ and $b_{n}$ such that

$$
\lim _{n \rightarrow \infty} P\left(M_{n} \leq a_{n} x+b_{n}\right)=\lim _{n \rightarrow \infty} F^{n}\left(a_{n} x+b_{n}\right)=G_{\gamma}(x),
$$

where $\gamma \in \mathbb{R}$ is referred to as the extreme value index. It is known that $G_{\gamma}(x)$ belongs to one of the following three classes:

$$
\begin{array}{lll}
\text { Type I Gumbel: } & \Lambda(x)=\exp \{-\exp (-x)\}, & x \in \mathbb{R} \text { for } \gamma=0 ; \\
\text { Type II Fréchet: } & \Phi_{1 / \gamma}(x)=\left\{\begin{array}{ll}
0, & x<0 \\
\exp \left\{-x^{-1 / \gamma}\right\}, & x \geq 0
\end{array} \text { for } \gamma>0 ;\right. \\
\text { Type III Weibull: } & \Psi_{-1 / \gamma}(x)=\left\{\begin{array}{ll}
\exp \left\{-(-x)^{-1 / \gamma}\right\}, & x<0 \\
1, & x \geq 0
\end{array} \text { for } \gamma<0 .\right.
\end{array}
$$

Received November 20, 2010; Revised June 6, 2011.

2010 Mathematics Subject Classification. Primary 60G70; Secondary 62E20, 60F05.

Key words and phrases. convergence rate of moments, maximum, second order regular variation, second order von Mises condition. 
Let $f(x)=(1 /(-\log F))^{\leftarrow}(x)=\inf \{y: 1 /(-\log F) \geq x\}$ for $x \geq 1$. It is known that (1.1) holds if and only if for all $x>0$,

$$
\lim _{t \rightarrow \infty} \frac{f(t x)-f(t)}{a(t)}=\frac{x^{\gamma}-1}{\gamma},
$$

where the auxiliary function $a(t) \in R V_{\gamma}$, i.e., $a(t x) / a(t) \rightarrow x^{r}$ for $x>0$. Note that (1.2) means $f(t)$ is a general regular varying function, written as $f \in G R V(\gamma, a)$. For more properties of general regular varying functions, see Geluk and de Haan [6]. Moreover, if $\gamma>0$, then (1.2) is equivalent to

$$
\lim _{t \rightarrow \infty} \frac{f(t x)}{f(t)}=x^{\gamma}, \quad x>0 .
$$

The auxiliary function $a(t) \sim \gamma f(t)$. For the case $\gamma<0, f(\infty)=x_{0}<\infty$ and (1.2) is equivalent to

$$
\lim _{t \rightarrow \infty} \frac{f(\infty)-f(t x)}{f(\infty)-f(t)}=x^{\gamma}, \quad x>0 .
$$

The auxiliary function $a(t) \sim-\gamma\{f(\infty)-f(t)\}$. For the case of $\gamma=0$, (1.2) implies that $f$ is a $\Pi$ regular varying function, i.e.,

$$
\lim _{t \rightarrow \infty} \frac{f(t x)-f(t)}{a(t)}=\log x, \quad x>0 .
$$

Convergence rates related to (1.1) are interesting in theoretical and empirical analysis. Hall [8] and Hall and Wellner [9] considered convergence rates of the extremes of normal and exponential distributions to their ultimate extreme value distribution $\Lambda(x)$. de Haan and Resnick [3] studied the convergence rates of (1.1) measured by the uniform metric and the total variation distance under a second von Mises condition to be mentioned later. Gomes and de Haan [7] and Kaufmann [10] considered some penultimate approximations under some second order regular varying conditions. Cheng and Jiang [1] derived Edgeworth expansions for the distribution of normalized maxima under some weak assumptions. It is well known that the weak convergence of a sequence of random variables does not imply convergence of the moments, see Resnick [14, pages 75-77] for the extremes. Liu and Peng [11] considered convergence rates of conditional moments studied by Geluk [5] and Peng et al. [12] under some additional assumptions. For the convergence of moments of normalized maxima, Pickands [13] showed that for given additional assumptions,

$$
\lim _{n \rightarrow \infty} E\left(a_{n}^{-1}\left(M_{n}-b_{n}\right)\right)^{k}=\int_{-\infty}^{\infty} x^{k} d G(x)
$$

holds for some integer $k$. We cite Pickands' results here as a proposition.

Proposition 1.1. For an extreme value distribution $G$, suppose that $F \in$ $D(G)$. 
(i) For $G=\Lambda$, set $b_{n}=(1 / 1-F)^{\leftarrow}(n)$ and $a_{n}=g\left(b_{n}\right)$, where $g(t) \sim$ $\int_{t}^{x_{0}}(1-F(s)) d s /(1-F(t))$ and $x_{0}$ denotes the right end point of $F$. If there is an integer $k>0$ such that $\int_{-\infty}^{0}|x|^{k} d F(x)<\infty$, then

$$
\lim _{n \rightarrow \infty} E\left(\left(M_{n}-b_{n}\right) / a_{n}\right)^{k}=\int_{-\infty}^{\infty} x^{k} d \Lambda(x)=(-1)^{k} \Gamma^{(k)}(1),
$$

where $\Gamma^{(k)}(1)$ is the $k$ th derivative of the gamma function at $x=1$.

(ii) For $G=\Phi_{1 / \gamma}$, set $a_{n}=(1 /(1-F))^{\leftarrow}(n)=\inf \{y: 1 /(1-F(y))>n\}$ and $b_{n}=0$. If there is an integer $0<k<1 / \gamma$ such that $\int_{-\infty}^{0}|x|^{k} d F(x)<\infty$, then

$$
\lim _{n \rightarrow \infty} E\left(M_{n} / a_{n}\right)^{k}=\int_{0}^{\infty} x^{k} d \Phi_{1 / \gamma}(x)=\Gamma(1-k \gamma),
$$

where $\Gamma(\cdot)$ denotes the gamma function.

(iii) For $G=\Psi_{-1 / \gamma}$, set $a_{n}=x_{0}-(1 / 1-F)^{\leftarrow}(n)$ and $b_{n}=x_{0}$, where $x_{0}$ denotes the right endpoint of $F$. If there is an integer $k>0$ such that $\int_{-\infty}^{x_{0}}|x|^{k} d F(x)<\infty$, then

$$
\lim _{n \rightarrow \infty} E\left(\left(M_{n}-x_{0}\right) / a_{n}\right)^{k}=\int_{-\infty}^{0} x^{k} d \Psi_{-1 / \gamma}(x)=(-1)^{k} \Gamma(1-k \gamma),
$$

where $\Gamma(\cdot)$ denotes the gamma function.

Remark 1. For any sequences $\alpha_{n}$ and $\beta_{n}$ such that $\alpha_{n} / a_{n} \rightarrow a>0$ and $\left(\beta_{n}-b_{n}\right) / a_{n} \rightarrow b \in \mathbb{R}$, Proposition 1.1 also holds with some linear transform of the extreme value distribution, i.e., with $G(x)$ replaced by $G(a x+b)$. For the special case $a=1$ and $b=0$, see Exercise 2.1.2 of Resnick [14].

The aim of this note is to consider the convergence rate of the moments of extremes. In order to obtain the desired results, we need to impose convergence rates on the general regular variation, or the regular variation and the $\Pi$ regular varying function, i.e., we need the convergence rates of (1.2)-(1.5). In general, we use the second order regular varying function widely used in extreme value theory. The general second order regular varying function has been studied by de Haan and Stadtmüller [4], i.e.,

Proposition 1.2. Suppose there exist a positive function a $(t)$, a function $A(t)$ with constant sign near infinity, satisfying $A(t) \rightarrow 0$ as $t \rightarrow \infty$ and $|A| \in R V_{\rho}$ with $\rho \leq 0$, and a function $K(x)$ that is not a multiple of $\left(x^{\gamma}-1\right) / \gamma$, such that

$$
\lim _{t \rightarrow \infty} \frac{1}{A(t)}\left\{\frac{f(t x)-f(t)}{a(t)}-\frac{x^{\gamma}-1}{\gamma}\right\}=K(x), \quad \forall x \in \mathbb{R}^{+} .
$$

Then

$$
K(x)=c_{1} \int_{1}^{x} s^{\gamma-1} \int_{1}^{s} u^{\rho-1} d u d s+c_{2} \int_{1}^{x} s^{\gamma+\rho-1} d s
$$


where $c_{1}$ and $c_{2}$ are constants. By a perfect choice of $a(t)$ and $A(t), K(x)$ can take the form

$$
K(x)=K_{\gamma, \rho}(x):=\left\{\begin{array}{l}
\frac{\log ^{2} x}{2} \quad \text { for } \quad \gamma=0=\rho ; \\
\frac{x^{\gamma} \log x}{\gamma} \quad \text { for } \quad \rho=0 \neq \gamma ; \\
\frac{x^{\gamma+\rho}-1}{\gamma+\rho} \quad \text { for } \quad \rho<0 .
\end{array}\right.
$$

For details, see Theorem 1 of de Haan and Stadtmüller [4], and Theorem 2.3.3 and Corollary 2.3.4 of de Haan and Ferreira [2].

The following simple second order regular varying conditions may be useful in this note. The first condition is the so-called second order regular variation.

Condition 1. Suppose there exists measurable function $A(t)$ with constant sign near infinity and $A(t) \rightarrow 0$ as $t \rightarrow \infty$ such that

$$
\lim _{t \rightarrow \infty} \frac{\frac{f(t x)}{f(t)}-x^{\gamma}}{A(t)}=x^{\gamma} \frac{x^{\rho}-1}{\rho}=: \varphi(x)
$$

for all $x>0$, where $\left(x^{\rho}-1\right) / \rho=: \log x$ if $\rho=0$. It is known that (1.7) implies $A(t) \in R V_{\rho}, \rho \leq 0$.

For the second order regular varying function, the following inequality holds.

Lemma 1.1. Assume that (1.7) holds for $f(t)$. Then there exists $C(t) \sim$ $t^{-\gamma} f(t) A(t) \in R V_{\rho}$ such that for positive $\varepsilon, \delta$ and $x>0$,

$$
\left|\frac{f(t x) / f(t)-x^{\gamma}}{C(t) t^{\gamma} / f(t)}-x^{\gamma} \frac{x^{\rho}-1}{\rho}\right| \leq \varepsilon x^{\gamma+\rho} \max \left(x^{\delta}, x^{-\delta}\right)
$$

for $\min (t, t x)>t_{0}(\varepsilon, \delta)$.

Proof. Clearly by (1.7),

$$
\frac{f(t x)(t x)^{-\gamma}-t^{-\gamma} f(t)}{A(t) t^{-\gamma} f(t)} \rightarrow \frac{x^{\rho}-1}{\rho}
$$

for all $x>0$ as $t \rightarrow \infty$. This implies $t^{-\gamma} f(t) \in G R V\left(\rho, A(t) t^{-\gamma} f(t)\right)$. So, by Theorem B.2.18 in de Haan and Ferreira [2], there exists $C(t) \sim A(t) t^{-\gamma} f(t)$ such that

$$
\left|\frac{f(t x)(t x)^{-\gamma}-t^{-\gamma} f(t)}{C(t)}-\frac{x^{\rho}-1}{\rho}\right| \leq \varepsilon x^{\gamma+\rho} \max \left(x^{\delta}, x^{-\delta}\right)
$$

for $\min (t, t x)>t_{0}(\varepsilon, \delta)$. The result follows.

The following is the second order regular variation condition on $\Pi$ regular varying functions. 
Condition 2. Suppose that there exists $A(t)$ with constant sign near infinity and $A(t) \rightarrow 0$ as $t \rightarrow \infty$ such that

$$
\lim _{t \rightarrow \infty} \frac{\frac{f(t x)-f(t)}{a(t)}-\log x}{A(t)}=\psi(x)
$$

for all $x>0$, where

$$
\psi(x)= \begin{cases}\frac{1}{2} \log ^{2} x, & \text { if } \quad \rho=0 ; \\ \frac{x^{\rho}-1}{\rho}, & \text { if } \quad \rho \neq 0 .\end{cases}
$$

It is known that (1.8) implies $A(t) \in R V_{\rho}, \rho \leq 0$.

For the second order regular variation of the $\Pi$ regular varying function, the following inequality holds.

Lemma 1.2. Suppose that (1.8) holds. Then there exist functions $a_{t}$ and $A_{1}(t)$ satisfying

$$
A_{1}(t) \sim A(t), \quad a(t) / a_{1}(t)-1=o\left(A_{1}(t)\right)
$$

and, for positive $\delta, \varepsilon$, there exists $t_{0}=t_{0}(\varepsilon, \delta)>0$,

$$
\left|\frac{\frac{f(t x)-f(t)}{a_{1}(t)}-\log x}{A_{1}(t)}-\psi(x)\right| \leq \varepsilon \max \left(x^{\rho+\delta}, x^{\rho-\delta}\right)
$$

for all $x>0$ as $\min (t, t x)>t_{0}$.

Proof. This is a special case of Theorem 2.3.6 in de Haan and Ferreira [2].

If $f(x)$ is twice differentiable, then the following second order von Mises condition defined by de Haan and Resnick [3] can be used to analyze convergence rates.

Definition 1.1. A function $f:(0, \infty) \mapsto \mathbb{R}$ satisfies a second-order von Mises condition with first-order parameter $\gamma \in \mathbb{R}$ and second-order parameter $\rho \leq 0$ if $f$ is twice differentiable, $f^{\prime}$ is eventually positive and the function

$$
B(t)=\frac{t f^{\prime \prime}(t)}{f^{\prime}(t)}-\gamma+1
$$

has constant sign near infinity and satisfies $\lim _{t \rightarrow \infty} B(t)=0$ and $|B| \in R V_{\rho}$. We write $f \in 2$ vonMises $(\gamma, \rho)$.

For the second-order von Mises function, the following results due to de Haan and Resnick [3] hold.

Lemma 1.3. Suppose $f \in 2$ vonMises $(\gamma, \rho)$. Then both

$$
\lim _{t \rightarrow \infty} \frac{f(t x)-f(t)}{t f^{\prime}(t)}=\frac{x^{\gamma}-1}{\gamma}
$$


and

$$
\frac{f^{\prime}(t x) / f^{\prime}(t)-x^{\gamma-1}}{B(t)} \rightarrow H^{\prime}(x)
$$

hold. Furthermore, for $\gamma \geq 0$,

$$
\frac{\frac{f(t x)-f(t)}{t f^{\prime}(t)}-\frac{x^{\gamma}-1}{\gamma}}{B(t)} \rightarrow H(x)
$$

and, for $\gamma<0, f(\infty):=\lim _{t \rightarrow \infty} f(t)<\infty$ and

$$
\frac{\frac{f(t x)-f(\infty)-\gamma^{-1} t f^{\prime}(t)}{t f^{\prime}(t)}-\frac{x^{\gamma}-1}{\gamma}}{B(t)} \rightarrow H(x),
$$

where

$$
H(x)=: H_{\gamma, \rho}(x)=\left\{\begin{array}{cl}
\int_{1}^{x} s^{\gamma-1} \int_{1}^{s} u^{\rho-1} d u d s & \text { for } \gamma \geq 0 \\
-\int_{x}^{\infty} s^{\gamma-1} \int_{1}^{s} u^{\rho-1} d u d s & \text { for } \gamma<0 .
\end{array}\right.
$$

Proof. See Theorem 2.1 of de Haan and Resnick [3].

Lemma 1.4. Suppose $f \in 2$ vonMises $(\gamma, \rho)$. Then, for given $\varepsilon>0$, there exists $t_{0}=t_{0}(\varepsilon)$ such that if $\min (t, t x)>t_{0}$, we have

$$
(1-\varepsilon) \exp (-\varepsilon|\log x|) \leq \frac{f^{\prime}(t x) / f^{\prime}(t)-x^{\gamma-1}}{B(t) H^{\prime}(x)} \leq(1+\varepsilon) \exp (\varepsilon|\log x|) .
$$

Furthermore, for $\gamma \geq 0$,

$$
\begin{aligned}
(1-\varepsilon) \exp (-\varepsilon|\log x|) & \leq \frac{[f(t x)-f(t)] / t f^{\prime}(t)-\left(x^{\gamma}-1\right) / \gamma}{B(t) H(x)} \\
& \leq(1+\varepsilon) \exp (\varepsilon|\log x|)
\end{aligned}
$$

and, for $\gamma<0$,

$$
\begin{aligned}
(1-\varepsilon) \exp (-\varepsilon|\log x|) & \leq \frac{\left[f(t x)-f(\infty)-\gamma^{-1} t f^{\prime}(t)\right] / t f^{\prime}(t)-\left(x^{\gamma}-1\right) / \gamma}{B(t) H(x)} \\
& \leq(1+\varepsilon) \exp (\varepsilon|\log x|) .
\end{aligned}
$$

Proof. See Theorem 2.3 of de Haan and Resnick [3].

The following result is immediate from Lemma 1.4.

Lemma 1.5. Suppose $f \in 2$ vonMises $(\gamma, \rho)$ and set $v(t)=f(\exp (t))$. For given $\varepsilon>0$, there exists $n_{0}=n_{0}(\varepsilon)$ such that for $n \geq n_{0}$ and $\log n+u \geq \log n_{0}$,

$$
\frac{P_{n}(u)}{B(n) H(\exp (u))}=1+\delta_{\varepsilon}^{(1)}(u) \quad \text { and } \quad \frac{P_{n}^{\prime}(u)}{B(n) H^{\prime}(\exp (u))}=1+\delta_{\varepsilon}^{(2)}(u)
$$

with

$$
(1-\varepsilon) \exp (-\varepsilon|u|) \leq 1+\delta_{\varepsilon}^{(i)}(u) \leq(1+\varepsilon) \exp (\varepsilon|u|), \quad i=1,2,
$$


where $P_{n}(u)=\left\{v(u+\log n)-b_{n}\right\} / v^{\prime}(\log n)-\{\exp (\gamma u)-1\} / \gamma$ with

$$
b_{n}=\left\{\begin{aligned}
v(\log n) & \text { for } \quad \gamma \geq 0 \\
v(\infty)+\gamma^{-1} v^{\prime}(\log n) & \text { for } \quad \gamma<0
\end{aligned}\right.
$$

\section{Main results}

In this section, we give the main results. The proofs are deferred to Section 3.

We need some notations. Set

$$
f(t)=(-1 / \log F)^{\leftarrow}(t) \text { and } v(t)=(-\log (-\log F))^{\leftarrow}(t),
$$

so that $v(t)=f(\exp (t))$. If $f(t)$ is strictly increasing for large $t$, then $U(t)=$ $-\log (-\log F(t))$ is also strictly increasing for $t$ in the neighborhood of $x_{0}=$ $f(\infty)$, the right end point of $F$. Note that $a_{n} x+b_{n} \rightarrow x_{0}$ if $F \in D(G)$. Define a new variable $u$ such that $-u=\log n-U\left(a_{n} x+b_{n}\right)$, implying $a_{n} x+b_{n}=$ $v(u+\log n)$ for large $n$ and $u \rightarrow \log (-\log G(x))=u_{0}$ say, as $n \rightarrow \infty$.

Theorem 2.1. Suppose that $F \in D(G)$ and $f(t)=(-1 / \log F)^{\leftarrow}(t)$ is strictly increasing for large $t$.

(i) Suppose $G=\Lambda$ and (1.8) holds. Assume further that (1.6) holds with $a_{n}=a(n), b_{n}=f(n)$,

$$
\frac{v(\log n+u)-v\left(\log n+u_{0}\right)}{A(n) a(n)} \rightarrow 0
$$

and

$$
\frac{u_{0}-u}{A(n)} \rightarrow 0
$$

as $n \rightarrow \infty$. Then

$$
\frac{1}{A(n)}\left(E\left(\left(M_{n}-b_{n}\right) / a_{n}\right)^{k}-\int_{-\infty}^{\infty} x^{k} d \Lambda(x)\right) \rightarrow \int_{-\infty}^{\infty} k x^{k-1} \psi(\exp (x)) d \Lambda(x) .
$$

(ii) Suppose $G=\Phi_{1 / \gamma}$ and (1.6) holds with $a_{n}=f(n), b_{n}=0$. Assume further that (1.7) holds,

$$
\frac{v(\log n+u)-v\left(\log n+u_{0}\right)}{A(n) a(n)} \rightarrow 0
$$

and

$$
\frac{u_{0}-u}{A(n)} \rightarrow 0
$$

as $n \rightarrow \infty$. Then

$$
\frac{1}{A(n)}\left(E\left(M_{n} / a_{n}\right)^{k}-\int_{0}^{\infty} x^{k} d \Phi_{1 / \gamma}(x)\right) \rightarrow \int_{0}^{\infty} k x^{k}\left(\frac{x^{\rho / \gamma}-1}{\rho}\right) d \Phi_{1 / \gamma}(x) .
$$


(iii) Suppose $G=\Psi_{-1 / \gamma}$ and (1.6) holds with $a_{n}=f(\infty)-f(n), b_{n}=$ $f(\infty)=x_{0}<\infty$. Assume further that (1.7) holds for $f^{*}(t)=f(\infty)-f(t)$,

$$
\frac{v(\log n+u)-v\left(\log n+u_{0}\right)}{A(n)[f(\infty)-v(\log n)]} \rightarrow 0
$$

and

as $n \rightarrow \infty$. Then

$$
\frac{u_{0}-u}{A(n)} \rightarrow 0
$$

$$
\begin{aligned}
& \frac{1}{A(n)}\left(E\left(\left(M_{n}-x_{0}\right) / a_{n}\right)^{k}-\int_{-\infty}^{0} x^{k} d \Psi_{-1 / \gamma}(x)\right) \\
\rightarrow & \int_{-\infty}^{0} k x^{k}\left(\frac{(-x)^{\rho / \gamma}-1}{\rho}\right) d \Psi_{-1 / \gamma}(x) .
\end{aligned}
$$

The second order von Mises condition allows us to treat the convergence rates for the moments of extremes uniformly.

Theorem 2.2. For $F \in D(G)$, set $f(t)=(1 /-\log F)^{\leftarrow}(t)$ and assume that $f \in 2$ von $M i s e(\gamma, \rho)$ with auxiliary function $B(t)=t f^{\prime \prime}(t) / f^{\prime}(t)-\gamma+1$. Let $v(t)=f(\exp (t)), a_{n}=v^{\prime}(\log n)$ and

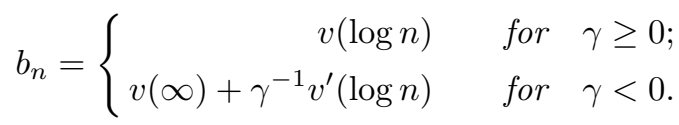

Assume further that (1.6) holds for given $a_{n}$ and $b_{n}$. Then

$$
\begin{aligned}
& \frac{1}{B(n)}\left(E\left(a_{n}^{-1}\left(M_{n}-b_{n}\right)\right)^{k}-\int_{\gamma x+1>0} x^{k} d G_{\gamma}(x)\right) \\
\rightarrow & \int_{\gamma x+1>0} k x^{k-1} H\left((\gamma x+1)^{1 / \gamma}\right) d G_{\gamma}(x) .
\end{aligned}
$$

The following is a necessary condition for the convergence rate of the moments of extremes.

Theorem 2.3. Under the conditions of Proposition 1.1, assume that (1.1) holds and $f(t)$ is strictly increasing. If there exists $A^{*}(t)$ such that $A^{*}(t) \rightarrow 0$ as $t \rightarrow \infty$, and

$$
\frac{1}{A^{*}(n)}\left(E\left(a_{n}^{-1}\left(M_{n}-b_{n}\right)\right)^{k}-\int_{-\infty}^{\infty} x^{k} d G(x)\right) \rightarrow c
$$

as $n \rightarrow \infty$, where $c \neq 0$, then $P_{n}(u) / A^{*}(n)$ does not approach zero uniformly with respect to $u$ as $n \rightarrow \infty$, where

$$
P_{n}(u)=\frac{f(n \exp (u))-b_{n}}{a_{n}}-\frac{\exp (\gamma u)-1}{\gamma} .
$$

Now we obtain unique convergence rates for the moments of extremes. 
Corollary 2.1. Suppose (2.1) holds for some $A^{*}(t) \rightarrow 0$ as $t \rightarrow \infty$. Then under the conditions of Theorem 2.1, we have

$$
A_{n} / A_{n}^{*} \rightarrow \kappa \neq 0
$$

as $n \rightarrow \infty$. Furthermore, for $G=\Lambda, G=\Phi_{1 / \gamma}$ and $G=\Psi_{-1 / \gamma}$, we have $P_{n}(u) / A^{*}(n)$ approaching $\kappa \psi(\exp (u)), \kappa \varphi(\exp (u))$ and $-\kappa \varphi(\exp (u))$, respectively.

Corollary 2.2. Suppose (2.1) holds for some $A^{*}(t) \rightarrow 0$ as $t \rightarrow \infty$. Then under the conditions of Theorem 2.2, we have

$$
A_{n} / A_{n}^{*} \rightarrow \kappa \neq 0
$$

as $n \rightarrow \infty$ and $P_{n}(u) / A^{*}(n)$ approaches $\kappa H(\exp (u))$.

\section{Proofs}

In this section, we use the notations defined previously.

Proof of Theorem 2.1. We consider the cases $G=\Lambda, G=\Phi_{1 / \gamma}$ and $G=\Psi_{-1 / \gamma}$ in turn.

(i) Note that $a_{n} x+b_{n}=v(u+\log n)$ for large $n$. We have

$$
\begin{aligned}
& E\left(\left(M_{n}-b_{n}\right) / a_{n}\right)^{k}-\int_{-\infty}^{\infty} x^{k} d \Lambda(x) \\
= & \int_{-\infty}^{\infty} x^{k} d F^{n}\left(a_{n} x+b_{n}\right)-\int_{-\infty}^{\infty} x^{k} d \Lambda(x) \\
= & \int_{-\infty}^{\infty} x^{k} d \exp \left\{-\exp \left[\log n-U\left(a_{n} x+b_{n}\right)\right]\right\}-\int_{-\infty}^{\infty} x^{k} d \Lambda(x) \\
= & \int_{-\infty}^{\infty}\left(\frac{v(u+\log n)-b_{n}}{a_{n}}\right)^{k} d \Lambda(u)-\int_{-\infty}^{\infty} u^{k} d \Lambda(u) .
\end{aligned}
$$

Put

$$
P_{n}(u)=\frac{v(u+\log n)-b_{n}}{a_{n}}-u
$$

and note that $b_{n}=f(n)=v(\log n), a_{n}=a(n)$. By (1.8), we have

$$
\begin{aligned}
P_{n}(u) & =\frac{v(\log n+u)-v(\log n)}{a(n)}-u \\
& =\frac{v(\log n+u)-v\left(\log n+u_{0}\right)}{a(n)}+\frac{v\left(\log n+u_{0}\right)-v(\log n)}{a(n)}-u \\
& \sim \frac{v(\log n+u)-v\left(\log n+u_{0}\right)}{a(n)}+u_{0}-u+A(n) \psi\left(\exp \left(u_{0}\right)\right)
\end{aligned}
$$


for large $n$. Note that (3.1) also implies that $P_{n}(u) \rightarrow 0$ as $n \rightarrow \infty$. Combining with (3.1) and Lemma 1.2, we obtain

$$
\begin{aligned}
& \frac{1}{A(n)}\left(E\left(\left(M_{n}-b_{n}\right) / a_{n}\right)^{k}-\int_{-\infty}^{\infty} x^{k} d \Lambda(x)\right) \\
\sim & k \int_{-\infty}^{\infty} \frac{P_{n}(u)}{A(n)} u^{k-1} d \Lambda(u) \\
\rightarrow & k \int_{-\infty}^{\infty} \psi\left(\exp \left(u_{0}\right)\right) u_{0}^{k-1} d \Lambda\left(u_{0}\right)
\end{aligned}
$$

as $n \rightarrow \infty$, where the final step follows by dominated convergence theorem. The desired result follows for $G=\Lambda$.

(ii) Note that $a_{n}=f(n)=v(\log n)$ and $b_{n}=0$. Put

$$
P_{n}(u)=\frac{v(u+\log n)}{v(\log n)}-\exp (\gamma u), \quad s(u)=\exp (\gamma u) .
$$

So, by (1.7),

$$
\begin{aligned}
& P_{n}(u) \\
= & \frac{v\left(\log n+u_{0}\right)}{v(\log n)} \frac{v(\log n+u)}{v\left(\log n+u_{0}\right)}-\exp (\gamma u) \\
= & \left\{\frac{v\left(\log n+u_{0}\right)}{v(\log n)}-\exp \left(\gamma u_{0}\right)+\exp \left(\gamma u_{0}\right)\right\}\left\{\frac{v(\log n+u)}{v\left(\log n+u_{0}\right)}-1+1\right\}-\exp (\gamma u) \\
\sim & A(n) \varphi\left(\exp \left(u_{0}\right)\right)+\left\{A(n) \varphi\left(\exp \left(u_{0}\right)\right)+\exp \left(\gamma u_{0}\right)\right\} \frac{v(\log n+u)-v\left(\log n+u_{0}\right)}{v\left(\log n+u_{0}\right)} \\
& +\exp \left(\gamma u_{0}\right)-\exp (\gamma u)
\end{aligned}
$$

as $n \rightarrow \infty$. By Lemma 1.1 and arguments similar to those of part (i), we obtain

$$
\begin{aligned}
& \frac{1}{A(n)}\left(E\left(M_{n} / a_{n}\right)^{k}-\int_{-\infty}^{\infty} x^{k} d \Phi_{1 / \gamma}(x)\right) \\
= & \int_{-\infty}^{\infty}\left\{\left(P_{n}(u)+s(u)\right)^{k}-s^{k}(u)\right\} d \Lambda(u) / A(n) \\
\sim & k \int_{-\infty}^{\infty} \frac{P_{n}(u)}{A(n)} s^{k-1}(u) d \Lambda(u) \\
\rightarrow & k \int_{-\infty}^{\infty} \varphi\left(\exp \left(u_{0}\right)\right) s^{k-1}\left(u_{0}\right) d \Lambda\left(u_{0}\right)
\end{aligned}
$$

as $n \rightarrow \infty$, where the final step follows by dominated convergence theorem. Noting $\Lambda\left(\log x^{1 / \gamma}\right)=\Phi_{1 / \gamma}(x)$ as $x>0$, the desired result follows for $G=\Phi_{1 / \gamma}$.

(iii) Let $f^{*}(t)=f(\infty)-f(t)$, and note $a_{n}=f^{*}(n)=v(\infty)-v(\log n)$, $b_{n}=x_{0}=v(\infty)$. Set

$$
P_{n}(u)=\frac{v(u+\log n)-x_{0}}{x_{0}-v(\log n)}+\exp (\gamma u), \quad s(u)=-\exp (\gamma u) .
$$


By (1.7), one has

$$
\begin{aligned}
P_{n}(u)= & \frac{v\left(u_{0}+\log n\right)-x_{0}}{x_{0}-v(\log n)}+\exp \left(\gamma u_{0}\right)+\frac{v(u+\log n)-v\left(u_{0}+\log n\right)}{x_{0}-v(\log n)} \\
& +\exp (\gamma u)-\exp \left(\gamma u_{0}\right) \\
\sim & -A(n) \varphi\left(\exp \left(u_{0}\right)\right)+\frac{v(u+\log n)-v\left(u_{0}+\log n\right)}{x_{0}-v(\log n)} \\
& +\exp (\gamma u)-\exp \left(\gamma u_{0}\right)
\end{aligned}
$$

as $n \rightarrow \infty$. Combining with Lemma 1.1, we obtain

$$
\begin{aligned}
& \frac{1}{A(n)}\left(E\left(\left(M_{n}-x_{0}\right) / a_{n}\right)^{k}-\int_{-\infty}^{\infty} x^{k} d \Psi_{-1 / \gamma}(x)\right) \\
= & \frac{1}{A(n)} \int_{-\infty}^{\infty}\left\{\left(P_{n}(u)+s(u)\right)^{k}-s^{k}(u)\right\} d \Lambda(u) \\
\sim & k \int_{-\infty}^{\infty} \frac{P_{n}(u)}{A(n)} s^{k-1}(u) d \Lambda(u) \\
\rightarrow & -k \int_{-\infty}^{\infty} \varphi\left(\exp \left(u_{0}\right)\right) s^{k-1}\left(u_{0}\right) d \Lambda\left(u_{0}\right),
\end{aligned}
$$

where the final step follows by dominated convergence theorem. Noting that $\Lambda\left(\log (-x)^{1 / \gamma}\right)=\Psi_{-1 / \gamma}(x)$ as $x<0$, we obtain the desired result for $G=$ $\Psi_{-1 / \gamma}$. The proof is complete.

Proof of Theorem 2.2. Firstly, note that for $a_{n}=v^{\prime}(\log n)$ and

$$
b_{n}=\left\{\begin{array}{cl}
v(\log n) & \text { for } \quad \gamma \geq 0 \\
v(\infty)+\gamma^{-1} v^{\prime}(\log n) & \text { for } \quad \gamma<0
\end{array}\right.
$$

one has

$$
P\left(M_{n} \leq a_{n} x+b_{n}\right) \rightarrow G_{\gamma}(x) .
$$

Define a new variable $u$ such that $-u=\log n+\log \left(-\log F\left(a_{n} x+b_{n}\right)\right)$, implying that $a_{n} x+b_{n}=v(u+\log n)$. Clearly,

$$
\begin{aligned}
& E\left(\left(M_{n}-b_{n}\right) / a_{n}\right)^{k}-\int_{-\infty}^{\infty} x^{k} d G_{\gamma}(x) \\
= & \int_{-\infty}^{\infty} x^{k} d \exp \left\{-\exp \left[\log n+\log \left(-\log F\left(a_{n} x+b_{n}\right)\right)\right]\right\} \\
& -\int_{-\infty}^{\infty} x^{k} d \Lambda\left(\gamma^{-1} \log (1+\gamma x)\right) \\
= & \int_{-\infty}^{\infty}\left(\frac{v(u+\log n)-b_{n}}{a_{n}}\right)^{k} d \Lambda(u)-\int_{-\infty}^{\infty}\left(\frac{\exp (\gamma u)-1}{\gamma}\right)^{k} d \Lambda(u) .
\end{aligned}
$$


Define $s(u)=\{\exp (\gamma u)-1\} / \gamma$ and

$$
P_{n}(u)=\left\{v(u+\log n)-b_{n}\right\} / v^{\prime}(\log n)-\{\exp (\gamma u)-1\} / \gamma .
$$

By Lemma 1.5, we obtain

$$
\begin{aligned}
& \frac{1}{B(n)}\left(E\left(\left(M_{n}-b_{n}\right) / a_{n}\right)^{k}-\int_{-\infty}^{\infty} x^{k} d G_{\gamma}(x)\right) \\
\rightarrow & \int_{-\infty}^{\infty} k s^{k-1}(u) H(\exp (u)) d \Lambda(u)
\end{aligned}
$$

as $n \rightarrow \infty$. The proof is complete upon noting $G_{\gamma}(x)=\Lambda\left(\gamma^{-1} \log (1+\gamma x)\right)$.

Proof of Theorem 2.3. Firstly, note (1.1) implies

$$
\frac{v(x+\log n)-b_{n}}{a_{n}} \rightarrow \frac{\exp (\gamma x)-1}{\gamma}
$$

locally uniformly for $x$ as $n \rightarrow \infty$, where $v(t)=f(\exp (t))$. Define a new variable $u$ such that $-u=\log n+\log \left(-\log F\left(a_{n} x+b_{n}\right)\right)$. The strictly increasing $f(t)$ implies $a_{n} x+b_{n}=v(\log n+u)$. Let

$$
P_{n}(u)=\frac{v(u+\log n)-b_{n}}{a_{n}}-\frac{\exp (\gamma u)-1}{\gamma}
$$

and $s(u)=\{\exp (\gamma u)-1\} / \gamma$. By arguments similar to those of Theorem 2.2, we obtain

$$
\begin{aligned}
& E\left(\left(M_{n}-b_{n}\right) / a_{n}\right)^{k}-\int_{-\infty}^{\infty} x^{k} d G_{\gamma}(x) \\
= & \int_{-\infty}^{\infty}\left(\frac{v(u+\log n)-b_{n}}{a_{n}}\right)^{k} d \Lambda(u)-\int_{-\infty}^{\infty}\left(\frac{\exp (\gamma u)-1}{\gamma}\right)^{k} d \Lambda(u) \\
= & k \int_{-\infty}^{\infty} \int_{0}^{1}\left(s(u)+y P_{n}(u)\right)^{k-1} P_{n}(u) d y d \Lambda(u) .
\end{aligned}
$$

So,

$$
\begin{aligned}
& \frac{1}{A^{*}(n)}\left(E\left(\left(M_{n}-b_{n}\right) / a_{n}\right)^{k}-\int_{-\infty}^{\infty} x^{k} d G_{\gamma}(x)\right) \\
= & k \int_{-\infty}^{\infty}\left(\int_{0}^{1}\left(s(u)+y P_{n}(u)\right)^{k-1} d y\right) \frac{P_{n}(u)}{A^{*}(n)} d \Lambda(u) \\
= & k \sum_{l=0}^{k-1} \int_{0}^{1} y^{k-l-1} d y \int_{-\infty}^{\infty} \frac{s^{l}(u) P_{n}^{k-l}(u)}{A^{*}(n)} d \Lambda(u) \\
= & k \sum_{l=0}^{k-1} \frac{1}{k-l} \int_{-\infty}^{\infty} \frac{s^{l}(u) P_{n}^{k-l}(u)}{A^{*}(n)} d \Lambda(u) \rightarrow c \neq 0 .
\end{aligned}
$$


Now suppose $P_{n}(u) / A^{*}(n) \rightarrow 0$ uniformly with respect to $u$. Then since $\int_{-\infty}^{\infty} s^{l}(u) d \Lambda(u)$ is finite for all $l$ each integral in (3.2) approaches zero by the dominated convergence theorem, a contradiction.

\section{Examples}

For simplicity, the three examples given in this section satisfy the conditions of Theorem 2.2. Note $F \in D\left(G_{\gamma}\right)$ if and only if

$$
\frac{f^{\prime}(t x)}{f^{\prime}(t)} \rightarrow x^{\gamma-1}
$$

as $t \rightarrow \infty$ (de Haan and Resnick, [3]), where $f(t)=(1 /(-\log F))^{\leftarrow}(t)$. The three examples are based on the exponential, uniform and Pareto distributions.

Example 4.1. Consider the standard exponential distribution with $F(x)=$ $1-\exp (-x), x>0$. One can check that

$$
f(t)=-\log \{1-\exp (-1 / t)\}, f^{\prime}(t)=t^{-2}\{\exp (1 / t)-1\}^{-1}, \frac{f^{\prime}(t x)}{f^{\prime}(t)} \rightarrow x^{-1} .
$$

So, $F \in D\left(G_{0}\right)$, where $G_{0}(x)=\Lambda(x)$ and $\gamma=0$. Furthermore,

$$
\begin{aligned}
& f^{\prime \prime}(t)=-2 t^{-3}\{\exp (1 / t)-1\}^{-1}+t^{-4}\{\exp (1 / t)-1\}^{-2} \exp (1 / t), \\
& B(t)=\frac{t f^{\prime \prime}(t)}{f^{\prime}(t)}-0+1=t^{-1}\{\exp (1 / t)-1\}^{-1} \exp (1 / t)-1 .
\end{aligned}
$$

For $x>0$, rewrite

$$
\frac{B\left((x y)^{-1}\right)}{B\left(y^{-1}\right)}=\frac{[\exp (y)-1][x y \exp (x y)-\exp (x y)+1]}{[\exp (x y)-1][y \exp (y)-\exp (y)+1]} .
$$

Note that

$$
\lim _{y \downarrow 0} \frac{\exp (y)-1}{\exp (x y)-1}=x^{-1},
$$

and

$$
\lim _{y \downarrow 0} \frac{x y \exp (x y)-\exp (x y)+1}{y \exp (y)-\exp (y)+1}=x^{2} .
$$

So,

$$
\lim _{y \downarrow 0} \frac{B\left((x y)^{-1}\right)}{B\left(y^{-1}\right)}=x,
$$

i.e.,

$$
\lim _{t \rightarrow \infty} \frac{B(t x)}{B(t)}=x^{-1}
$$

for $x>0$, implying $B(t) \in R V_{-1}$ and $\rho=-1$. So, by Theorem 2.2 , we obtain

$$
\frac{1}{B_{n}}\left(E\left(a_{n}^{-1}\left(M_{n}-b_{n}\right)\right)^{k}-\int_{-\infty}^{\infty} x^{k} d G_{0}(x)\right)
$$




$$
\rightarrow \int_{-\infty}^{\infty} k x^{k-1}[x+\exp (-x)-1] d G_{0}(x)
$$

as $n \rightarrow \infty$, where $a_{n}=n f^{\prime}(n)=n^{-1}\{\exp (1 / n)-1\}^{-1}$ and $b_{n}=f(n)=$ $-\log \{1-\exp (-1 / n)\}$.

Example 4.2. Consider the uniform distribution with $F(x)=x$ for $0<x<1$. So, the right end point of $F$ is $x_{0}=1$. One can check that

$$
f(t)=\exp (-1 / t), \quad f^{\prime}(t)=t^{-2} \exp (-1 / t)
$$

and

$$
\frac{f^{\prime}(t x)}{f^{\prime}(t)} \rightarrow x^{-2}=x^{-1-1}
$$

So, $F \in D\left(G_{-1}\right)$ with $\gamma=-1$. One can also check that

$$
B(t)=\frac{t f^{\prime \prime}(t)}{f^{\prime}(t)}-(-1)+1=t^{-1},
$$

implying $B(t) \in R V_{-1}$ and $\rho=-1$. So, by Theorem 2.2 , we have

$$
\begin{aligned}
& n\left(E\left(a_{n}^{-1}\left(M_{n}-b_{n}\right)\right)^{k}-\int_{0}^{1} x^{k} d G_{-1}(x)\right) \\
\rightarrow & \int_{0}^{1} k x^{k-1}\left(\frac{1}{2}(1-x)^{2}-(1-x)\right) d G_{-1}(x)
\end{aligned}
$$

as $n \rightarrow \infty$, where $a_{n}=n f^{\prime}(n)=n^{-1} \exp (-1 / n)$ and $b_{n}=x_{0}+\gamma^{-1} n f^{\prime}(n)=$ $1-n^{-1} \exp (-1 / n)$.

Example 4.3. Consider the Pareto distribution with $F(x)=1-x^{-1 / \gamma}, x \geq 1$, where $0<\gamma<1 / 2$. Obviously, $F \in D\left(G_{\gamma}\right)$ and

$$
\begin{aligned}
f(t) & =\left\{1-\exp \left(-t^{-1}\right)\right\}^{-\gamma} \\
f^{\prime}(t) & =\gamma t^{-2} \exp \left(-t^{-1}\right)\left\{1-\exp \left(-t^{-1}\right)\right\}^{-\gamma-1} \\
f^{\prime \prime}(t) & =\gamma t^{-3} \exp \left(-t^{-1}\right) \frac{-2+t^{-1}+(\gamma+1) t^{-1} \exp \left(-t^{-1}\right)\left[1-\exp \left(-t^{-1}\right)\right]^{-1}}{\left\{1-\exp \left(-t^{-1}\right)\right\}^{\gamma+1}} .
\end{aligned}
$$

One can check that

$$
\begin{aligned}
B(t) & =\frac{t f^{\prime \prime}(t)}{f^{\prime}(t)}-\gamma+1 \\
& =-2+t^{-1}+(\gamma+1) \exp \left(-t^{-1}\right)\left\{t\left[1-\exp \left(-t^{-1}\right)\right]\right\}^{-1}-\gamma+1 \\
& =-2+t^{-1}+(\gamma+1)\left[1-t^{-1}+\cdots\right]\left[1-\frac{1}{2} t^{-1}+\cdots\right]^{-1}-\gamma+1 \\
& =-2+t^{-1}+(\gamma+1)\left[1-t^{-1}+\cdots\right]\left[1+\frac{1}{2} t^{-1}+\cdots\right]-\gamma+1 \\
& =t^{-1}+(\gamma+1)\left(\frac{1}{2} t^{-1}-t^{-1}\right)+\cdots
\end{aligned}
$$




$$
\sim \frac{1-\gamma}{2} t^{-1}
$$

implying that $B(t) \in R V_{-1}$ and $\rho=-1$. So, by Theorem 2.2 , we obtain

$$
\begin{aligned}
& \frac{1}{B(n)}\left(E\left(a_{n}^{-1}\left(M_{n}-b_{n}\right)\right)^{k}-\int_{1+\gamma x>0} x^{k} d G_{\gamma}(x)\right) \\
\rightarrow & \int_{1+\gamma x>0} k x^{k-1}\left(x-\frac{y^{\gamma-1}-1}{\gamma-1}\right) d G_{\gamma}(x)
\end{aligned}
$$

as $n \rightarrow \infty$, where $y=(1+\gamma x)^{1 / \gamma}, a_{n}=n\{1-\exp (-1 / n)\}^{-\gamma}$ and $b_{n}=$ $\{1-\exp (-1 / n)\}^{-\gamma}$.

Acknowledgments. The authors would like to thank the editor and the referee for careful reading and for their comments which greatly improved the paper.

\section{References}

[1] S. H. Cheng and C. G. Jiang, The Edgeworth expansion for distributions of extreme values, Sci. China Ser. A 44 (2001), no. 4, 427-437.

[2] L. de Haan and A. Ferreira, Extreme Value Theory: An Introduction, Springer-Verlag, New York, 2006.

[3] L. de Haan and S. I. Resnick, Second order regular variation and rates of convergence in extreme value theory, Ann. Probab. 24 (1996), no. 1, 97-124.

[4] L. de Haan and U. Stadtmüller, Generalized regular variation of second order, J. Austral. Math. Soc. Ser. A 61 (1996), no. 3, 381-395.

[5] J. L. Geluk, On the domain of attraction of $\exp (\exp (-x))$, Statist. Probab. Lett. 31 (1996), no. 2, 91-95.

[6] J. L. Geluk and L. de Haan, Regular variation, Extensions and Tauberian Theorems, CWI Tract, 40. Stichting Mathematisch Centrum, Centrum voor Wiskunde en Informatica, Amsterdam, 1987.

[7] M. I. Gomes and L. de Haan, Approximation by penultimate extreme value distributions, Extremes 2 (1999), no. 1, 71-85.

[8] P. Hall, On the rate of convergence of normal extremes, J. Appl. Probab. 16 (1979), no. 2, 433-439.

[9] W. J. Hall and J. A. Wellner, The rate of convergence in law of the maximum of an exponential sample, Statist. Neerlandica 33 (1979), no. 3, 151-154.

[10] E. Kaufmann, Penultimate approximations in extreme value theory, Extremes 3 (2000), no. $1,39-55$.

[11] K. Liu and Z. Peng, The convergence rates of conditional moments, Journal of Southwest University (Natural Science) 29 (2007), 5-8.

[12] Z. Peng, M. Liu, and S. Nadarajah, Conditions based on conditional moment for maxstable limit laws, Extremes 11 (2008), no. 4, 329-337.

[13] J. Pickands, Moment convergence of sample extremes, Ann. Math. Statist. 39 (1968), 881-889.

[14] S. I. Resnick, Extreme Values, Regular Variation and Point Processes, Springer-Verlag, New York, 1987. 
ZuOXIANG PENG

School of Mathematics and Statistics

SOUTHWEST UNIVERSITY

Chongqing, 400715, P. R. China

SARALEes NADARAJAH

SCHOOL OF Mathematics

UNIVERSITY OF MANCHESTER

Manchester, United Kingdom

E-mail address: saralees.nadarajah@manchester.ac.uk 